\title{
CONSULTA DE PRÉ-NATAL DE ENFERMAGEM: CUIDADO ALÉM DOS ASPECTOS FISIOLÓGICOS
}

\author{
Regiane Aparecida TEIXEIRA ${ }^{1}$ \\ Gleidson Brandão OSELAME ${ }^{2}$ \\ Denecir de Almeida DUTRA ${ }^{3}$ \\ Elia Machado de OLIVEIRA ${ }^{4}$ \\ Eduardo Borba NEVES
}

\begin{abstract}
${ }^{1}$ Enfermeira. Centro Universitário Campos de Andrade. Contato: regi_bem_loca@hotmail.com.
${ }^{2}$ Enfermeiro. Mestre em Engenharia Biomédica. Programa de Pós Graduação em Engenharia Biomédica (UTFPR). Centro Universitário Campos de Andrade. Contato: gleidsonoselame@gmail.com.

${ }^{3}$ Geógrafo. Doutor em Geografia. Centro Universitário Campos de Andrade. Contato: denecir.dutra@ terra.com.br.

${ }^{4}$ Enfermeira. Mestre em Cirurgia. Centro Universitário Campos de Andrade. Contato: elia561 @ hotmail.com .

${ }^{5}$ Fisioterapeuta. Doutor em Engenharia Biomédica. Programa de Pós Graduação em Engenharia Biomédica (UTFPR). Centro Universitário Campos de Andrade. Contato: borbaneves@ hotmail.com.
\end{abstract}

\section{Recebido em: 0705/2015 - Aprovado em: 18/09/2015 - Disponibilizado em: 30/10/2015}

\begin{abstract}
RESUMO
Objetivou-se descrever a percepção das gestantes em relação à abordagem do enfermeiro durante a consulta de prénatal e identificar seus sentimentos emergidos nesse processo. Tratou-se de uma pesquisa qualitativa de caráter exploratório, realizada em uma unidade de saúde no município de São José dos Pinhais - PR. A amostra foi composta por 22 gestantes com média de idade de 26,5 anos. Os resultados foram categorizados em: Caracterização das mulheres; Cuidado pré-natal: Acolhimento a gestante e a família; Gravidez Planejada; Histórico de aborto; Sexualidade e Gravidez; Mudanças no corpo feminino durante a gravidez; Relação entre profissionais e pacientes; Cuidado integral a gestante: Medos; Sentimentos e Instabilidade emocional. Entre os vários os sentimentos despertados pelas gestantes, o medo do parto esteve em evidência e foi associado ao fato de ( $\mathrm{n}=13 ; 59,09 \%)$ estarem do terceiro trimestre de gestação. A assistência prestada pelos profissionais em relação à abertura para o dialogo durante as consultas foi unânime entre as gestantes, porém $(n=3 ; 13,63 \%)$ não se sentiam a vontade para expressar suas dúvidas pela falta de vinculo com o profissional que as atende. Os dados revelam que são muitos os medos e sentimentos envolvidos no processo gestacional sendo de grande importância a criação do vinculo.

Palavras - chave: Gestantes. Relações materno-fetais. Pessoal de Saúde.

\section{NURSING PRENATAL CONSULTATION: CAUTION IN ADDITION TO THE PHYSIOLOGICAL ASPECTS}

\begin{abstract}
The objective was to describe the perception of pregnant women in relation to the approach nurse during prenatal consultation and identify their feelings emerged in this process. This was a qualitative study of exploratory character, performed in a health care facility in São José dos Pinhais - PR. The sample consisted of 22 pregnant women with a mean age of 26.5 years. The results were categorized into: Women characterization; Prenatal care: Home to the mother and family; Planned pregnancy; Abortion History; Sexuality and Pregnancy; Changes in the female body during pregnancy; Relationship between professionals and patients; Comprehensive care to pregnant women: Fears; Feelings and emotional instability. Among the various feelings awakened by pregnant women, birth fear was in evidence and was associated with the fact $(\mathrm{n}=13 ; 59.09 \%)$ are the third trimester of pregnancy. The assistance provided by professionals on the opening for dialogue during consultations was unanimous among pregnant women, however $(\mathrm{n}=$ $3 ; 13.63 \%$ ) did not feel free to express their doubts by the lack of link with the professional that meets. The data shows that there are many fears and feelings involved in the gestation process is of great importance to creation of bond Key words: Pregnant Women. Maternal-Fetal Relations. Health Personnel.
\end{abstract}




\section{INTRODUÇÃO}

No âmbito da Estratégia de Saúde da família (ESF) é de competência da equipe multidisciplinar o acolhimento a todo cliente, especialmente a gestante. A assistência no cuidado durante a gravidez inclui a prevenção de doenças e agravos, a promoção de saúde e problemas ocorridos durante a gestação (DUARTE, 2006).

Apesar das inúmeras mudanças físicas, sociais e emocionais ocorridas no período gestacional, é importante deixar claro que a gestação é um fenômeno fisiológico o qual deve ser visto como uma experiência de vida saudável (DE AQUINO CUNHA et al., 2009).

Tendo o acolhimento como uma política de humanização, baseado em ações que favorecem a relação de confiança entre o usuário para com a equipe de saúde, o profissional passa a ter um papel de ouvinte onde permite que a gestante se expresse sem medo de julgamentos. Na prática isso ocorre mediante atitudes simples como, por exemplo, chamar o usuário pelo nome ou apenas apresentar-se (DUARTE, 2006).

A intervenção de enfermagem iniciase antes mesmo da confirmação da gravidez, com uma simples curiosidade e/ou dúvida de saber se está grávida ou não, após a comprovação da gestação através de exames diagnósticos de dosagem de hormônio gonadotrófico coriônico na urina ou no sangue, inicia-se o pré-natal. Compete ao enfermeiro realizar orientações sobre a importância do pré-natal, cadastrar a gestante no Sistema de Acompanhamento do Programa de Humanização no Pré-Natal e Nascimento (SisPreNatal) e fornecer a carteirinha da gestante devidamente preenchida, além de prescrever medicamentos padronizados (SERRUYA, CECATTI e DO LAGO, 2004; DE AQUINO CUNHA et al., 2009).

São inúmeras as atribuições do enfermeiro dentro da equipe de atenção básica, porém a consulta de pré-natal de baixo risco destaca-se pela responsabilidade como binômio mãe-filho, o que também envolve a família (JÚNIOR et al., 2013).

Sabe-se que o vínculo é uma ferramenta essencial para que se estabeleça um bom trabalho, pois é através dele que a gestante vai demonstrar sentimentos como de Ambivalência (querer e não querer a gravidez); Ansiedade e dúvidas sobre estar grávida ou não, uma vez que o feto não é concretamente sentido; Medo de abortar; Oscilações de humor e Instabilidade 
emocional (RIOS e VIEIRA, 2007). Desta forma o presente estudo teve por objetivo descrever a percepção das gestantes em relação à abordagem do enfermeiro durante a consulta de pré-natal e seus sentimentos emergidos neste processo.

\section{METODOLOGIA}

Tratou-se de uma pesquisa qualitativa de caráter exploratório, realizada em uma Unidade de Saúde localizada no município de São José dos Pinhais, região metropolitana de Curitiba, onde, atualmente é composta por quatro equipes de Estratégia de Saúde da Família (ESF) onde abrange cerca de 25.0000 habitantes(DOS SANTOS PEREIRA et al., 2013).

A coleta de dados foi realizada entre Agosto e Setembro de 2014 e utilizou como instrumentos o questionário socioeconômico composto por 12 questões e questionário com 20 perguntas abertas e fechadas a fim de identificar a sua percepção acerca da gestação.

Para compor a amostra, foram préestabelecidos critérios de inclusão para o estudo dentre os quais: ter acima de 18 anos, estar fazendo acompanhamento de pré-natal apenas na atenção de baixo-risco, concordar em participar e assinar o Termo de Consentimento Livre e Esclarecido.

Os critérios de exclusão para participação da pesquisa foram: transferência para acompanhamento privado e mudança de endereço. Após se estabelecer os critérios de inclusão/exclusão participaram do estudo 22 gestantes.

A interpretação dos dados foi realizada pela técnica da análise de conteúdo, método que consiste em identificar, dentro de cada eixo temático, categorias analíticas e posteriormente, alocar as falas dentro das mesmas, o que se chama de categorização final em unidades de análise (NEVES e MELLO, 2009; TABORDA et al., 2014).

As gestantes foram identificadas através da letra $\mathrm{G}$ seguida por um algarismo arábico, de acordo com os relatos, ou seja, G1, G2, G3... A fim de preservar o sigilo das mesmas e atender a Resolução 466/2012 que trata da proteção dos participantes das pesquisas cientificas envolvendo seres humanos do Conselho Nacional de Saúde, a pesquisa foi autoriza pelo estabelecimento de saúde e aprovado pelo Comitê de Ética em Pesquisa do Centro Universitário Campos de Andrade com o protocolo de $n^{\circ}$ 288709. 
RESULTADOS

A idade das mulheres variou de 18 a 43 anos com média de 26,5 anos, sendo a faixa etária predominante entre 19 e 25 anos ( $\mathrm{n}=9 ; 40,90 \%)$. As variáveis de perfil socioeconômico analisadas são detalhadas na Tabela 1.

Tabela 1. Identificação das características demográficas e socioeconômicas de gestantes em acompanhamento de pré-natal de baixo risco. São José dos Pinhais, 2014

\begin{tabular}{l|c|c}
\hline \multicolumn{1}{c|}{ Variáveis } & n & \% \\
\hline Etnia & 11 & 50 \\
Branca & 10 & 45,45 \\
Parda & 1 & 4,54 \\
Amarela & 14 & \\
Religião & 7 & 63,63 \\
Católico & 1 & 31,81 \\
Protestante ou Evangélico & & 4,54 \\
Umbanda ou Candomblé & 3 & 13,63 \\
Idade Gestacional & 6 & 27,27 \\
Entre 4 e 13 semanas & 13 & 59,09 \\
Entre 17 e 26 semanas & & \\
Entre 30 e 39 semanas & 5 & 22,72 \\
Estado Civil & 16 & 72,72 \\
Solteiro & 1 & 4,54 \\
Casada mora com um companheiro & 7 & \\
Divorciada & 2 & 31,81 \\
Número de Filhos & 2 & 9,09 \\
Um & 2 & 9,09 \\
Dois & 9 & 9,09 \\
Três & & 40,90 \\
Quatro ou mais & 7 & \\
Nenhum & 15 & 61,81 \\
Tipo de Residência & & \\
Própria & 7 & 31,81 \\
Alugada & 68,18 \\
Escolaridade & 6 & 27,27 \\
Ensino Fundamental completo & 4 & 18,18 \\
Ensino Fundamental Incompleto & 3 & 13,63 \\
Ensino Médio completo & 2 & 9,09 \\
Ensino Médio Incompleto & 11 & 50 \\
Ensino superior & 1 & 45,45 \\
Renda Familiar & & 4,54 \\
De 1 a 2 salários mínimos & & \\
De 2 a 5 salários mínimos & & \\
De 5 a 10 salários mínimos & & \\
\hline & & \\
\hline
\end{tabular}

\section{DISCUSSÃO}

Da análise dos dados surgiram as categorias: Caracterização das mulheres; Cuidado pré-natal: Acolhimento a gestante e a família; Mudanças no corpo feminino durante a gravidez; Gravidez Planejada; Histórico de aborto; Relação 
entre profissional e paciente; Sexualidade e gravidez; Cuidado integral a gestante: Medos; Sentimentos; Instabilidade emocional.

\section{Caracterização das mulheres}

Com relação ao perfil da amostra estudada, observa-se que as gestantes desta região estão sendo mães mais novas em relação a média de idade para o Sul do Brasil que é de 27,4 anos (PICCININI et al., 2012; ISOBE et al., 2013).

Em relação ao número de filhos $(\mathrm{n}=9$; $40,90 \%)$ serão mães pela primeira vez, $(n=7$; $31,81 \%$ ) tem apenas um filho. Resultados semelhantes são evidenciados no senso de 2010 realizado pelo Instituo Brasileiro de Geografia e Estatística (IBGE) que é de 1,9 filhos por família (Instituto Brasileiro de Geografia e Estatística (IBGE). Censo demográfico 2010: resultados gerais da amostra, 2010).

No que diz respeito ao nível de escolaridade elas variaram entre, ensino fundamental completo $(\mathrm{n}=7 ; 31,81 \%)$ e incompleto $(\mathrm{n}=6 ; 27,27 \%)$, ensino médio completo $(\mathrm{n}=4 ; 18,18 \%)$ e incompleto $(\mathrm{n}=3$; $13,63 \%)$, além de curso superior completo $(n=2$; 9,09\%). Tal fato assemelha-se ao estudo de Xavier et al.,(XAVIER et al., 2013) onde foram avaliadas 1462 mulheres e cerca de $60 \%$ possuíam no máximo o primeiro grau completo, fator preocupante, pois a menor escolaridade esta diretamente ligada com a maior dificuldade de compreensão (ISOBE et al., 2013).

$\mathrm{O}$ perfil econômico da população analisada é caracterizado como de baixa renda, ou seja, pertencentes à classe menos favorecida $(\mathrm{n}=11$; $50 \%$ ) ganham de um a dois salários mínimos, associa-se a isto o comprometimento da renda mensal com moradia, pois $(\mathrm{n}=15 ; 68,18 \%)$ das famílias não tem residência própria, e pagam aluguel.

Estes dados mostram o potencial de risco dos sujeitos envolvidos, pois eventos da vida estressante ocasionado pela dificuldade financeira implicam significativamente em agravos na saúde mental destas gestantes(TABORDA et al., 2014). No entanto o fato de $(n=16 ; 72,72 \%)$ serem casadas, diminui esta probabilidade, pois é sabido que $o$ apoio familiar principalmente do companheiro é de grande importância (THIENGO et al., 2011).

\section{Cuidado pré-natal: Acolhimento a gestante e a família}

Observa-se na população analisada que a maioria destaca como importante a participação do parceiro nas consultas $(\mathrm{n}=19 ; 86,36 \%)$, porém quando comparados a real participação dos mesmos, apenas $(n=9 ; 40,90 \%)$ participam efetivamente. Um estudo realizado recentemente aponta que o principal motivo da não participação 
do pai na consulta é o trabalho, alegando que o horário da mesma ocorre no período comercial, porém, apesar das dificuldades impostas é importante que o profissional estimule a participação de um acompanhante a esta gestante, durante o período pré-natal, parto, parto e pós parto, pois é de seu direito conforme preconiza a Lei $\mathrm{n}^{\mathrm{o}}$ 11.108, de sete de abril de 2005 (DE OLIVEIRA et al., 2009).

$\mathrm{O}$ acolhimento a gestante no seu contexto social é de grande importância, neste processo esta envolvida a família. Ao serem questionadas quanto à aceitação da gravidez perante os familiares a maioria das gestantes classificou como muito boa $(n=16 ; 72,72 \%)$ e as demais como boa $(n=6$; 27,27). Ao que se refere à aceitação perante o parceiro as mesmas variáveis estiveram entre: Ruim ( $\mathrm{n}=1 ; 4,54 \%)$; Regular ( $\mathrm{n}=2 ; 9,09 \%)$; Boa $(n=5 ; 22,72 \%)$; Muito Boa $(n=14 ; 63,63 \%)$.

Observa-se que a melhor aceitação foi dentre os demais familiares em relação aos companheiros, fatores emocionais e culturais podem estar associados a este resultado, pois o impacto da paternidade pode ser interpretado de maneiras distintas (DE OLIVEIRA et al., 2009).

\section{Mudanças no corpo feminino durante a gravidez}

São inúmeras as mudanças que ocorrem no corpo feminino durante a gestação, e muitas vezes elas podem ser vistas de forma negativa pelas mulheres devido a vários fatores como o medo de não voltar ao corpo anterior, de sentir- se indesejada, entre outros (PICCININI et al., 2012).

Quando questionadas $(\mathrm{n}=8 ; \quad 36,36 \%)$ responderam que se sentem incomodadas com mudanças estéticas dentre os relatos estão:

“Gorda, inchada, etc...” (G1)

“Um pouco só, saiu algumas manchas no meu rosto..." (G2)

"Aumentou meu quadril” (G3)

“Emagrecimento" (G4)

Por outro lado a maioria $(n=14,63,63 \%)$ não apresentou incomodo com essas mudanças, fator positivo, pois se observa que essas transformações não interferem na vida social das mesmas, pelo contrário sendo a maternidade vista de forma positiva, como o aumento dos seios associado ao aumento da beleza feminina (SHIMIZU e LIMA, 2009).

\section{Gravidez Planejada}

Nesta pesquisa, pode se observar um alto índice de gestações não planejadas $(\mathrm{n}=11 ; 50 \%)$, de forma semelhante ao observado no estudo realizado por Hernandez e Hutz(HERNANDEZ e HUTZ, 2008) onde foram pesquisadas 135 mulheres primigestas e dessas $42,2 \%$ engravidaram acidentalmente, fato freqüente não só em cidadãs 
brasileiras, mas também em norte americanas onde metade das gestações não são planejadas (LIMA E SILVA et al., 2011).

Dados como esses mostram a vulnerabilidade social da população em pesquisa, pois o planejamento da gravidez permite que a mulher adote hábitos de vida mais saudáveis como, por exemplo, parar de fumar antes mesmo de engravidar, sendo assim o trauma psicológico será menor, podendo associar a intenção de engravidar como uma mudança positiva no comportamento dessas mulheres (LIMA E SILVA et al., 2011). No estudo de Taborda et al., (TABORDA et al., 2014) observaram que os métodos contraceptivos eram conhecidos pelas mulheres, mas não utilizados na prevenção da gravidez indesejada.

Contudo, o não planejamento da gestação mostra não estar associado ao desejo de abortar, sentimento muitas vezes despertado num primeiro momento, pois $21(95,45 \%)$ das gestantes não manifestaram este desejo. Apenas uma $(n=1$; $4,54 \%$ ) manifestou. O relato abaixo descreve a motivação:

“Medo de reação da família." (G1)

\section{Histórico de aborto}

Segundo Ministério da Saúde aborto é a morte ou expulsão ovular ocorrida antes de 22 semanas ou quando o feto pesa menos de $500 \mathrm{~g}$ podendo ser espontâneo ou provocado, no caso das gestantes pesquisadas não foi especificada a forma de aborto e nem a quantidade por cada gestante (DOMINGUES et al., 2012).

O abortamento foi um episódio relativamente freqüente entre as grávidas desta região esteve entre não $(n=14 ; 63,63 \%)$ e $\operatorname{sim}(n=8$; $36,36 \%)$ já tiveram algum aborto, considerando que são vários os sentimentos que envolvem este processo como frustração, tristeza, medo, é de se esperar que em uma nova gestação estes sentimentos ainda estejam presentes e muitas vezes com maior intensidade. No estudo de Fernandes et al.,(FERNANDES et al., 2012) podemos observar estes sentimentos claramente entre os depoimentos, onde a grande maioria despertou a felicidade de se estar grávida, porém norteadas pelo receio de um novo aborto.

Dentro deste contexto é de grande importância o enfermeiro estar atento a estes dados não apenas como histórico obstétrico cumprindo protocolos estabelecidos, mas com uma visão ampla no processo de assistência.

\section{Relação entre profissional e paciente}

O compromisso da equipe de ESF inicia-se desde o desejo da mulher engravidar até o inicio do pré-natal propriamente dito, sendo o enfermeiro membro desta equipe é importante sua participação na assistência prestada à gestante. De acordo com a Lei do Exercício Profissional da Enfermagem, 
Decreto n. ${ }^{\circ}$ 94.406/87 e o Ministério da Saúde do Brasil, o pré-natal de baixo risco pode ser inteiramente acompanhado pelo enfermeiro. Neste contexto faz-se necessário que o profissional estabeleça uma relação de confiança com o paciente e a família envolvida neste processo (Conselho Federal de Enfermagem (COFEN). Decreto $\mathrm{N}^{\circ}$ 94.406/87. Regulamenta a Lei $\mathrm{n}^{\circ}$ 7.498, de 25 de junho de 1986, que dispõe sobre o exercício da Enfermagem, e dá outras providências. ).

Esta relação de confiança criada entre profissional e paciente é de grande importância para que se estabeleça um bom trabalho, pode-se observar na população analisada um alto índice ao que se refere a isso, pois $(n=21 ; 95,45 \%)$ descreveram manter uma relação de confiança com o profissional que a atende.

Quanto ao que diz respeito do vínculo do profissional/paciente onde questionou se elas sentem-se a vontade para expressar suas dúvidas $19(86,36 \%)$ responderam que sim se sentem a vontade e $(n=3 ; 13,63 \%)$ responderam que não. Alguns depoimentos das mulheres que destacaram o motivo:

\section{“Mudança de profissional.” (G1)}

"Mudaram bastante os profissionais que me atenderam, já consultei com quatro pessoas diferentes isso me deixa insegura." (G2)
Os depoimentos evidenciam que há uma falha na continuidade do atendimento à gestante, fator agravante, pois a mudança de profissional tem sido associada à má qualidade do pré-natal (VIELLAS et al., 2014).

O diálogo entre profissional e paciente é de grande importância para que se estabeleçam trocas de experiências, neste contexto a questão foi inânime entre as gestantes, pois todas responderam que o profissional oportuniza espaço durante a consulta para o dialogo e esclarecimento de dúvidas.

\section{Sexualidade e gravidez}

O desempenho sexual durante a gravidez pode variar bastante, dentre as pesquisadas metade $(\mathrm{n}=11 ; 50 \%)$ classificou como normal durante a gestação, $(n=3 ; 13,63 \%)$ como aumentada, e $(n=8$; 36,36) como diminuído, a diminuição da libido pode estar associada a vários fatores como, por exemplo, dor no ato sexual, indisposição e cansaço, porém aspectos culturais também podem estar envolvidos neste processo como o medo de machucar o bebê, sendo assim destaca-se a importância da orientação a esta gestante durante a consulta, porém com limitação visando o respeito a crenças e diferentes culturas (DA SILVA, 2012; PICCININI et al., 2012). 


\section{Cuidado integral a gestante: Medos;} Sentimentos; Instabilidade emocional

A descoberta da gravidez pode gerar vários tipos de emoções e o desejo da maternidade contribui para a ocorrência de sentimentos positivos como, por exemplo, o de Felicidade $(\mathrm{n}=16 ; 72,72 \%)$ que predominou neste sentido, porém percebem-se os sentimentos norteados por insegurança classificados pelo Medo ( $\mathrm{n}=3$; 13,63\%); Espanto ( $\mathrm{n}=2 ; 9,09 \%$ ); e Preocupação, a qual foi citada uma vez $(\mathrm{n}=1 ; 4,54 \%)$. No entanto sendo este um momento delicado de fase muitas vezes de aceitação, cabe ao profissional orientar esta gestante e neste momento criar o vinculo fazendo sua captação para o inicio do pré-natal como recomenda o Programa de Humanização no Pré- natal e Nascimento (CARDOSO, 2007).

A gravidez é um período de grandes mudanças fisiológicas, biológicas e psicológicas, comumente ocorrem oscilações de humor entre as gestantes, das 22 pesquisadas $(\mathrm{n}=14 ; 63,63 \%)$ delas apresentaram alguma alteração, o stress foi citado em pelo menos 4 dos depoimentos outras oscilações podem ser observadas a seguir:

"Sim. Muito estresse falta de paciência e muitos nervosos." (G1)

"Sim, tem dia que acordo chateada por nada outros dias feliz" (G2)

"Impaciência, alegria, medo, insegurança" (G3)

"Ansiedade, agitação" (G4)
"As vezes fico um pouco brava por minhas roupas não servirem em mim, mas logo passa"

"Estressada, sentimental, nervosa." (G5)

Os agentes estressores, ou seja, atitudes que desencadeiam o estresse como eventos do dia-a-dia podem provocar reações como o medo e a ansiedade. Fatores que propiciam a depressão pósparto, desta forma o profissional deve estar atento a estes comportamentos, este fato demonstra a importância de se quebrar barreiras na consulta com uma visão além das dimensões biológicas, desempenhando um papel de ouvinte sem julgamentos (SHIMIZU e LIMA, 2009; AIROSA e SILVA, 2013; DA ROCHA ARRAIS, ALVES MOURÃO e FRAGALLE, 2014).

A análise dos dados no quesito maior medo durante o período gestacional teve um aumento no percentual geral, pois se devem levar em consideração as gestantes que optaram por mais de uma resposta, dentre elas estão: Do parto $(n=10$; 45,45\%); De doenças no bebe ( $\mathrm{n}=8 ; 36,36 \%)$; De abortamento ( $\mathrm{n}=6 ; 27,27 \%)$.

$\mathrm{O}$ fato de grande parte das gestantes estarem no ultimo trimestre gestacional $(n=13$; $59,09 \%$ ) implicou no resultado onde citaram como maior medo o do parto, culturalmente sabe-se que as mulheres brasileiras diferentemente das americanas não se preparam para o parto, fatores como o medo da dor durante o parto ou traumas de 
partos anteriores podem contribuir para o aumento deste índice, porém medidas educativas durante o período pré- natal pode mudar esta realidade, sendo a falta de informação um agravante neste sentindo (DA ROCHA PEREIRA, FRANCO e BALDIN, 2011; LIMA E SILVA et al., 2011).

Ao avaliar o maior medo das gestantes após o nascimento do bebe pode-se observar que a questão saúde está presente na maioria dos relatos:

"Como vai ficar meu corpo e se ele vai nascer bem de saúde” (G1)

"Se tiver problema de saúde" (G3)

"De doenças" (G4)

"Se ele ou eu tenha alguma complicação pósparto (qualquer uma)" (G5)

"De não ser uma boa mãe” (G7)

"Com a saúde dele, quero muito que seja uma criança saudável” (G8)

As altas taxas de mortalidade neonatal e pós-natal divulgados pela mídia e infeliz realidade atual no Brasil implicam em depoimentos como os citados anteriormente, porém sabe-se que ao se estabelecer medidas de assistência adequadas e efetivas durante o período pré-natal, com um cuidado integral visando cumprir políticas de humanização no atendimento diminuem significativamente esses dados (GONÇALVES, CESAR e MENDOZA-SASSI, 2009; ANDREUCCI e CECATTI, 2011).

\section{CONSIDERAÇÕES FINAIS}

Constatou-se que grande parte das gestantes estava satisfeita com a consulta de pré-natal realizada pelo enfermeiro, porém faz-se necessário que se estabeleçam medidas para a continuidade no atendimento a gestante.

Observou-se que o medo do parto esteve em evidência entre as mulheres, medidas educativas devem ser implantadas com o objetivo de diminuir o impacto físico, mental e emocional no momento do parto, tanto em grupos específicos como em consultas individuais.

\section{REFERÊNCIAS}

AIROSA, S.; SILVA, I. Associação entre vinculação, ansiedade, depressão, stresse e suporte social na maternidade. Psicologia, Saúde \& Doenças, v. 14, n. 1, p. 64-77, 2013. ISSN 16450086.

ANDREUCCI, C. B.; CECATTI, J. G. Desempenho de indicadores de processo do Programa de Humanização do Pré-natal e Nascimento no Brasil: uma revisão sistemática Evaluation of the Program for Humanization of Prenatal and Childbirth Care in Brazil. Cad. saúde pública, v. 27, n. 6, p. 1053-1064, 2011.

CARDOSO, R. S. Programa de humanização no pré-natal e nascimento. 2007. Dissertaçã Programa de Pós-Graduação em Enfermage, Universidade Federal de Santa Catarina, Florianópolis.

Conselho Federal de Enfermagem (COFEN). Decreto $\mathrm{N}^{\circ}$ 94.406/87. Regulamenta a Lei $\mathrm{n}^{\circ}$ 7.498 , de 25 de junho de 1986, que dispõe sobre o 
exercício da Enfermagem, e dá outras providências. . Disponível em: < Disponível em: $<$ http://www.cofen.gov.br/decreto-n-

9440687_4173.html $>$. >. Acesso em: Acesso em 02 de novembro de 2014.

DA ROCHA ARRAIS, A.; ALVES MOURÃO, M.; FRAGALLE, B. O pré-natal psicológico como programa de prevenção à depressão pós-parto. Saúde e Sociedade, v. 23, n. 1, p. 251-264, 2014. ISSN 0104-1290.

DA ROCHA PEREIRA, R.; FRANCO, S. C.; BALDIN, N. Representações Sociais e decisões das gestantes sobre a parturição: protagonismo das mulheres. Saúde e Sociedade, v. 20, n. 3, p. 579589, 2011. ISSN 1984-0470.

DA SILVA, L. C. F. P. Corpo e sexualidade na gravidez. Rev Esc Enferm USP, v. 46, n. 3, p. 552-8, 2012.

DE AQUINO CUNHA, M. et al. Assistência prénatal: competências essenciais desempenhadas por enfermeiros. Escola Anna Nery Revista de Enfermagem, v. 13, n. 1, p. 145-153, 2009. ISSN 1414-8145.

DE OLIVEIRA, S. C. et al. A participação do homem/pai no acompanhamento da assistência prénatal. Cogitare Enfermagem, v. 14, n. 1, p. 73-8, 2009. ISSN 2176-9133.

DOMINGUES, R. M. S. M. et al. Avaliação da adequação da assistência pré-natal na rede SUS do Município do Rio de Janeiro, Brasil Adequacy of prenatal care in the National Health System in the city of Rio de Janeiro, Brazil. Cad. saúde pública, v. 28, n. 3, p. 425-437, 2012.

DOS SANTOS PEREIRA, D. D. et al. Análise da taxa de utilização e perda de vacinas no programa nacional de imunização. Cad. Saúde Colet, v. 21, n. 4, p. 420-4, 2013.
DUARTE, S. J. H. Assistência pré-natal no Programa Saúde da Família. Esc. Anna Nery Rev. Enferm, v. 10, n. 1, p. 121-126, 2006. ISSN 14148145.

FERNANDES, D. L. et al. Percepção de um grupo de gestantes detentoras de história de aborto em gestação anterior. Revista Brasileira de Ciências da Saúde-USCS, v. 10, n. 32, p. 47-53, 2012. ISSN 1678-054X.

GONÇALVES, C. V.; CESAR, J. A.; MENDOZA-SASSI, R. A. Qualidade e eqüidade na assistência à gestante: um estudo de base populacional no Sul do Brasil; Quality and equity in prenatal care: a population-based study in Southern Brazil. Cad. saúde pública, v. 25, n. 11, p. 2507-2516, 2009.

HERNANDEZ, J. A. E.; HUTZ, C. S. Gravidez do Primeiro Filho: Papéis Sexuais, Ajustamento Conjugal e Emocional1. Psicologia: Teoria e Pesquisa, v. 24, n. 2, p. 133-141, 2008.

Instituto Brasileiro de Geografia e Estatística (IBGE). Censo demográfico 2010: resultados gerais da amostra. Rio de Janeiro 2010.

ISOBE, M. T. et al. A influência da escolaridade na reprodutibilidade de um questionário quantitativo de frequência alimentar para gestantes; The influence of level of schooling on the reproducibility of a quantitative food frequency questionnaire among pregnant women. Rev. bras. saúde matern. infant, v. 13, n. 1, p. 23-28, 2013. ISSN 1519-3829.

JÚNIOR, A. R. F. et al. Vivência de adolescentes em atividade de promoção da saúde. Revista Brasileira de Enfermagem, v. 66, n. 4, p. 611614, 2013. ISSN 0034-7167.

LIMA E SILVA, J. et al. Assessment of psychosocial adaptation to pregnancy in brazilian pregnant women. Revista Brasileira de 
Ginecologia e Obstetrícia, v. 33, n. 8, p. 182-187, 2011. ISSN 0100-7203.

NEVES, E. B.; MELLO, M. G. S. O risco da profissão militar na cidade do Rio de Janeiro em" tempo de paz": a percepção da tropa. Cienc. saude colet., v. 14, n. 5, p. 1699-1707, 2009. ISSN 14138123.

PICCININI, C. A. et al. Percepções e sentimentos de gestantes sobre o pré-natal. Psicologia: Teoria e Pesquisa, v. 28, n. 1, p. 27-33, 2012. ISSN 1806-3446.

RIOS, C. T. F.; VIEIRA, N. F. C. Ações educativas no pré-natal: reflexão sobre a consulta de enfermagem como um espaço para educação em saúde. Ciência \& Saúde Coletiva, v. 12, n. 2, p. 477-486, 2007.

SERRUYA, S. J.; CECATTI, J. G.; DO LAGO, T. D. G. O Programa de Humanização no Pré-natal e Nascimento do Ministério da Saúde no Brasil: resultados iniciais The Brazilian Ministry of Health's Program for Humanization of Prenatal and Childbirth. Cad. saúde pública, v. 20, n. 5, p. 1281-1289, 2004.
SHIMIZU, H. E.; LIMA, M. G. The dimensions of prenatal care embodied in nursing consultation. Revista Brasileira de Enfermagem, v. 62, n. 3, p. 387-392, 2009. ISSN 0034-7167.

TABORDA, J. A. et al. Consequências da gravidez na adolescência para as meninas considerando-se as diferenças socioeconômicas entre elas. Cad. Saúde Colet, v. 22, n. 1, p. 16-24, 2014.

THIENGO, D. et al. Associação entre apoio social e depressão durante a gestação: uma revisão sistemática. Cad Saúde Colet, v. 19, n. 2, p. 12938, 2011.

VIELLAS, E. F. et al. Assistência pré-natal no Brasil. Cad. saúde pública, v. 30, n. supl. 1, p. 85100, 2014. ISSN 0102-311X.

XAVIER, R. B. et al. Risco reprodutivo e renda familiar: análise do perfil de gestantes. Ciênc saúde coletiva, v. 18, n. 4, p. 1161-71, 2013. 